\title{
ON BETA EXPANSIONS FOR PISOT NUMBERS
}

\author{
DAVID W. BOYD
}

\begin{abstract}
Given a number $\beta>1$, the beta-transformation $T=T_{\beta}$ is defined for $x \in[0,1]$ by $T x:=\beta x(\bmod 1)$. The number $\beta$ is said to be a betanumber if the orbit $\left\{T^{n}(1)\right\}$ is finite, hence eventually periodic. In this case $\beta$ is the root of a monic polynomial $R(x)$ with integer coefficients called the characteristic polynomial of $\beta$. If $P(x)$ is the minimal polynomial of $\beta$, then $R(x)=P(x) Q(x)$ for some polynomial $Q(x)$. It is the factor $Q(x)$ which concerns us here in case $\beta$ is a Pisot number. It is known that all Pisot numbers are beta-numbers, and it has often been asked whether $Q(x)$ must be cyclotomic in this case, particularly if $1<\beta<2$. We answer this question in the negative by an examination of the regular Pisot numbers associated with the smallest 8 limit points of the Pisot numbers, by an exhaustive enumeration of the irregular Pisot numbers in $[1,1.9324] \cup[1.9333,1.96]$ (an infinite set), by a search up to degree 50 in [1.9,2], to degree 60 in [1.96,2], and to degree 20 in $[2,2.2]$. We find the smallest counterexample, the counterexample of smallest degree, examples where $Q(x)$ is nonreciprocal, and examples where $Q(x)$ is reciprocal but noncyclotomic. We produce infinite sequences of these two types which converge to 2 from above, and infinite sequences of $\beta$ with $Q(x)$ nonreciprocal which converge to 2 from below and to the 6 th smallest limit point of the Pisot numbers from both sides. We conjecture that these are the only limit points of such numbers in $[1,2]$. The Pisot numbers for which $Q(x)$ is cyclotomic are related to an interesting closed set of numbers $\mathcal{F}$ introduced by Flatto, Lagarias and Poonen in connection with the zeta function of $T$. Our examples show that the set $S$ of Pisot numbers is not a subset of $\mathcal{F}$.
\end{abstract}

\section{INTRODUCTION}

For each real $\beta>1$ we define a mapping $T=T_{\beta}$ of the unit interval $[0,1]$ to itself by $T x:=\beta x(\bmod 1)$ for $x \in[0,1]$. Parry [21] defined $\beta$ to be a beta-number if the orbit $\left\{T^{n}(1)\right\}$ is finite. If $T^{n}(1)=0$ for some $n$, then $\beta$ is a simple beta-number. If $\beta$ is a beta-number which is not simple, then there is some smallest $m \geq 1$ (the preperiod length) and $p \geq 1$ (the period length) for which $T^{m+p}(1)=T^{m}(1)$. For a simple beta-number we define $m=0$ and $p$ to be the smallest integer with $T^{p}(1)=0$. (This is a natural convention as will be seen below.)

It is easy to see by induction that there are integers $c_{k}$ with $0 \leq c_{k}<\beta$ (the digits in the beta-expansion of 1 ) for which $T^{n}(1)=\beta^{n}-c_{1} \beta^{n-1}-\cdots-c_{n}$, so that

Received by the editor August 4, 1994 and, in revised form, February 13, 1995.

1991 Mathematics Subject Classification. Primary 11R06, 11K16; Secondary 11Y99.

Key words and phrases. Pisot numbers, beta-expansions, polynomials.

This research was supported by a grant from NSERC. 
$T^{n}(1)=P_{n}(\beta)$, where $P_{n}(x)=x^{n}-c_{1} x^{n-1}-\cdots-c_{n}$. Thus, if $\beta$ is a beta-number, then $\beta$ satisfies the polynomial equation $R(\beta)=0$, where

$$
R(x)= \begin{cases}P_{m+p}(x)-P_{m}(x) & \text { if } m>0 \\ P_{p}(x) & \text { if } m=0 .\end{cases}
$$

The polynomial $R$ is called the characteristic polynomial of $\beta$. Since $R(\beta)=0, \beta$ is an algebraic integer and hence is the root of a monic polynomial with integer coefficients of smallest degree $P(x)$, its minimal polynomial. Thus, $R(x)=P(x) Q(x)$ for some monic polynomial with integer coefficients $Q$. We will refer to $Q(x)$ as the complementary factor.

Parry [21] showed that the roots of $R$ other than $\beta$ lie in the disk $|z|<\min (2, \beta)$. This was improved to $|z| \leq(\sqrt{5}+1) / 2$ by Solomyak [24] and Flatto, Lagarias and Poonen [17] independently. In fact, Solomyak gives an exact description of a compact subset $\Phi$ of the plane which is the closure of the set of all of the roots other than $\beta$ of characteristic polynomials of beta-numbers. He shows, in fact, that $\Phi$ is the closure of the set of all roots other than $\beta$ of the minimal polynomials of beta-numbers.

This suggests that there will be some connection between beta-numbers and the sets $S$ and $T$ of Pisot and Salem numbers, which are defined to be those algebraic integers $\beta>1$ all of whose other conjugates lie in the open unit disk or closed unit disk, respectively (with at least one conjugate on the boundary of the disk in the case of Salem numbers). Indeed, Schmidt [23] showed that every Pisot number is a beta-number. This was also proved independently by Bertrand $[4,5]$. The idea behind the proof goes back to a paper of Gelfond [19]. Schmidt also showed that if the beta-expansion in base $\beta$ of every rational is periodic, then $\beta$ must be a Pisot or Salem number. However, it is not known whether all Salem numbers are beta-numbers, except for numbers of degree 4 [12].

An interesting connection between Pisot numbers and beta-expansions is a result of Berend and Frougny [2], which shows that normalization in base $\beta$ (conversion of an arbitrary expansion in base $\beta$ to the canonical beta-expansion) is computable by a finite automaton if and only if $\beta$ is a Pisot number. Frougny and Solomyak [18] have studied situations in which one can guarantee that a given Pisot number is a simple beta-number.

A question which naturally comes up in connection with the beta-expansions for Pisot numbers is the nature of the polynomials $Q$. We know that the roots of $Q$ must all be quite small - Solomyak's result gives very precise information about their location. By Kronecker's famous theorem, if all roots of $Q$ were to lie in the closed unit disk, then $Q$ would be a cyclotomic polynomial (here meaning only that all its roots are roots of unity, not necessarily that it is irreducible). In 1987, I. Katai asked the author whether $Q$ must always be cyclotomic in case $\beta$ is a Pisot number with $1<\beta<2$. Independently, in $1992 \mathrm{C}$. Frougny asked the same question without imposing this latter restriction.

This question also comes up in connection with the zeta-function $\zeta_{\beta}(z)$ of $T_{\beta}$, studied by Flatto, Lagarias and Poonen [17]. From equations (2.7) and (2.8) of [17], it is easy to show that

$$
\zeta_{\beta}(z)=\frac{1-z^{p}}{R^{*}(z)}
$$


where $R^{*}(z)=z^{m+p} R(1 / z)$ is the reciprocal polynomial of $R(z)$. Thus, the poles of the zeta-function are the reciprocals of the zeros of $R$ with the exception of $p$ th roots of unity. Note that (1.2) is valid whether or not beta is simple, giving some justification of the chosen definition of $m$ and $p$ for simple beta-numbers. A more fundamental reason for this choice is that, if one were to modify the definition of $T$ by choosing the fractional part to be in $(0,1]$ rather than the usual $[0,1)$, then $T^{n}(1)$ would be purely periodic with period $p$ exactly when $\beta$ is a simple beta-number.

The authors of [17] defined $M(\beta) \leq 1$ to be the absolute value of the second smallest pole of the zeta-function $(M(\beta)=1$ if there is no pole other than $1 / \beta$ in the unit disk), and showed that $M(\beta)$ is a continuous function of $\beta$. Hence, the set $\mathcal{F}=\{\beta \mid M(\beta)=1\}$ is a closed subset of the reals. (We should remark that $M(\beta)$ here has no relation with the Mahler measure of $\beta$ ). The only beta-numbers which can be in $\mathcal{F}$ must be Pisot or Salem numbers. They remark [17, p. 241] that they do not know of any Pisot number that is not in $\mathcal{F}$. Since the Pisot numbers which lie in $\mathcal{F}$ are exactly those for which $Q(x)$ is cyclotomic, our results provide many examples of such Pisot numbers.

In answer to Katai's question, we searched the list of Pisot numbers in $[1,1.932]$ compiled in connection with [7] and, after trying about 50 examples, found an example of a Pisot number $\beta=1.85855 \ldots$ of degree 23 with $(m, p)=(51,22)$ for which $Q(x)$ is nonreciprocal, hence noncyclotomic. (In this paper, a polynomial $Q(x)$ of degree $d$ is reciprocal if $x^{d} Q(1 / x)= \pm Q(x)$ and nonreciprocal otherwise). Later, after Frougny had raised the same question, we again looked at this table, finding two more examples, one of degree 26 with $(m, p)=(327,30)$ and one of degree 36 with $(m, p)=(1923,80)$, both with $Q(x)$ nonreciprocal (and both with $\beta=1.8667 \ldots$ to four decimal places).

These discoveries raised some natural questions: Are there examples for which $P(x)$ has smaller degree or for which $Q(x)$ has smaller degree? Is it possible for $Q(x)$ to be a reciprocal polynomial but not cyclotomic? How common is it for $Q(x)$ to be noncyclotomic? How are the $\beta$ for which $Q(x)$ is noncyclotomic distributed in the interval $[1,2]$ ?

This paper describes a systematic study of these questions which exploits the known structure of the Pisot numbers, in particular Amara's [1] identification of the limit points of the set $S \cap[1,2]$ and the algorithm developed in [7, 10 and 11]. We are able to obtain satisfactory answers to most of these questions. In particular, we find the smallest $\beta$ for which $Q(x)$ is nonreciprocal (equation (4.1)) and the smallest $\beta$ for which $Q(x)$ is reciprocal but noncyclotomic (equation (4.2)); this example has a factor $L(-x)$ where $L(x)$ is Lehmer's famous 10th-degree polynomial.

We find a $\beta<2$ of degree 7 with $P(x)=x^{7}-2 x^{5}-2 x^{4}-x-1$ for which $Q(x)$ is a nonreciprocal polynomial of degree 13 (given in equation (3.5)). By completely enumerating the $71 P(x)$ of degree at most 7 , we find that all $\beta$ of degree $\leq 6$ have cyclotomic $Q$, and the example mentioned in the previous sentence is the unique example of degree 7 for which $Q$ is noncyclotomic. The examples of Proposition 5.5 generalize this example.

As far as the degree of $Q$ itself is concerned, for $\beta<2$ the noncyclotomic $Q$ of smallest degree which was found is $Q(x)=x^{6}+x^{5}-x^{3}-x^{2}+1$ (equation (4.4)), which occurs for a $P(x)$ of degree 15 . This is probably the smallest degree attainable for noncyclotomic $Q$ with $\beta<2$, but of this we cannot be certain since it is conceivable that a smaller degree $Q(x)$ might occur for some unexamined $P(x)$ 
of large degree. On the other hand, for $\beta>2$, we can definitely find noncyclotomic complementary factors of smaller degree: in $[2,2.2]$ we find many examples for which $Q(x)=x^{3}-x^{2}+1$.

It was shown by Talmoudi $[25,26]$ that, for any $\delta>0$, the elements of $S \cap[1,2-\delta]$ fall into a countable number of families of regular Pisot numbers connected with the known limit points of $S \cap[1,2]$, and a finite set of irregular Pisot numbers. We showed in [10] how the finite set could be effectively enumerated. In the current study, for 34 of the 36 families of regular Pisot numbers associated with the smallest 8 limit points we have found that the polynomials $Q(x)$ are cyclotomic. For the two exceptional families, both associated with the limit point $\hat{\theta}_{2}^{\prime}=1.90516 \ldots$, a zero of $x^{4}-x^{3}-2 x^{2}+1$, one quarter of the $Q(x)$ are nonreciprocal. We conjecture that $Q(x)$ will be cyclotomic for all the remaining regular Pisot numbers in $[1,2]$. This would imply that the only possible accumulation points in [1,2] of $\beta$ with $Q(x)$ noncyclotomic would be $\hat{\theta}_{2}^{\prime}$ and 2 .

For the irregular Pisot numbers in $[1,1.9324] \cup[1.9333,1.96]$ there were a reasonable number of noncyclotomic $Q(x)$, roughly $5 \%$ of the total studied. For the interval $[1.96,2]$, we did not attempt a complete enumeration (there are infinitely many limit points of $S$ in this interval) but restricted the degree of $\beta$ to be at most 60 ; for the interval $[2,2.2]$, we restricted the degree to be at most 20. For the Pisot numbers in $[2,2.2]$, of degree at most 20 , the percentage of noncyclotomic $Q(x)$ is over $50 \%$, although the number of these which are reciprocal is a small fraction of the total.

Our computations produced only 38 examples of $\beta<2$ for which $Q(x)$ is reciprocal but noncyclotomic. For $\beta$ of this type, the largest degree for $P(x)$ is 36 in spite of a search up to degree 60 . This perhaps suggests that there are only a finite number of examples of this type with $\beta<2$. On the other hand, Proposition 5.2, described next, shows that there are infinitely many such examples in any interval $[2,2+\delta]$ with $\delta>0$.

Finally, suggested by the computations described in $\S \S 3$ and 4 , we produce six interesting sequences of Pisot numbers $\beta_{k} \rightarrow 2$. In each of these, $\beta_{k}$ is the root of a polynomial of the form $f\left(x, x^{k}\right)$, where $f(x, y)$ is a polynomial in two variables. Five of these examples are described in Propositions 5.1 to 5.5. The examples of Propositions 5.1 and 5.2 have $\beta_{k}>2$. For the first of these (Proposition 5.1), the complementary factors are the simple nonreciprocal trinomials $x^{k+1}-x^{k}+1$. For the second (Proposition 5.2) the complementary factors are the reciprocal polynomials $x^{2 k+2}-x^{2 k+1}+x^{k+1}-x+1$, which are noncyclotomic for sufficiently large $k$.

The sequences of Propositions 5.3 to 5.5 have $\beta_{k}<2$, and the complementary factor is nonreciprocal in each case. These examples are considerably more complicated than those of Propositions 5.1 and 5.2. The simplest $Q(x)$ found here is $x^{4 k}+x^{k}-1$ for the even terms with $k \geq 4$ of the sequence of Proposition 5.4. We have not found a similar sequence with $\beta_{k} \uparrow 2$ for which the complementary factor is reciprocal but noncyclotomic. As mentioned above, the computations described here suggest that the set of Pisot numbers in $[1,2]$ with this property may be finite.

In the examples described in Propositions 5.1 to 5.5, the preperiod length $m$ and period length $p$ increase linearly with $k$ and the expansions follow a recognizable pattern which can be proved to hold for all $k \geq k_{0}$, where $k_{0}$ is given explicitly. On the other hand, for the sixth example, which is presented in Remark 5.4 and Table 2 , although $\beta_{k}<2$ satisfies a polynomial of the same general form as those 
in Propositions 5.3 to 5.5, the values of $m$ and $p$ appear to grow much more rapidly with $k$ and no clear pattern is evident. This indicates that there is no obvious generalization of Propositions 5.1 to 5.5 to families of the form $f\left(x, x^{k}\right)$, even when it can be proved that these polynomials define Pisot numbers for sufficiently large $k$.

\section{The Basic Structure of $S \cap[1,2]$}

Recall that a real number $\beta>1$ is a Pisot number (or Pisot-Vijayaraghavan number) if it is an algebraic integer all of whose conjugates other than $\beta$ lie in the open unit disk $\{z:|z|<1\}$. A famous result of Salem [22] is that the set of such numbers is a closed subset of the real line. The limit points of $S$ were characterized by Dufresnoy and Pisot [16] as follows: let $C(x)$ be the minimal polynomial of $\beta$; then $\beta$ is a limit point of $S$ if and only if there is a nonzero polynomial $A(x)$ with integer coefficients and $A(0) \neq 0$ such that $|A(x)| \leq|C(x)|$ on the unit circle $|x|=1$, with equality being attained only at a finite set of points. There will generally be more than one choice for $A(x)$ for a given $C(x)$.

Let us call the minimal polynomial of a Pisot number a Pisot polynomial. Associated with every limit point $\beta$ are two sequences of polynomials

$$
P_{n}(x)=x^{n} C(x) \pm A(x) .
$$

For all sufficiently large $n, P_{n}(x)$ has a unique root outside the unit circle and hence is the product of a Pisot polynomial and a cyclotomic polynomial. (For smaller $n$, $P_{n}(x)$ is cyclotomic). The cyclotomic factor must be a factor of the fixed Laurent polynomial $C(x) C(1 / x)-A(x) A(1 / x)$. We call the roots of the $P_{n}(x)$ the regular Pisot numbers determined by $\beta$.

Amara [1] determined the complete set of limit points of $S$ in $[1,2$ [ together with the associated polynomials $A(x)$. These fall into two infinite sequences $\left\{\phi_{r}\right\}$ and $\left\{\psi_{r}\right\}$, and one exceptional number $\hat{\theta}_{2}^{\prime}$. These have minimal polynomials, $C(x)$, given by

$$
\begin{gathered}
x^{r}(x-2)+(x-1) \text { for } \phi_{r}, \\
\left(x^{r+1}(x-2)+1\right) /(x-1)=x^{r+1}-x^{r}-x^{r-1}-\cdots-x-1 \text { for } \psi_{r},
\end{gathered}
$$

and

$$
x^{4}-x^{3}-2 x^{2}+1 \text { for } \hat{\theta}_{2}^{\prime} .
$$

The corresponding $A(x)$ are as follows:

$$
\begin{gathered}
x^{r}-x^{r-1}+1, \quad x^{r}-x+1 \text { and }\left(x^{r}+1\right)(x-1) \text { for } \phi_{r}, \\
x^{r+1}-1 \text { and }\left(x^{r}+1\right) /(x+1) \text { for } \psi_{r},
\end{gathered}
$$

and

$$
x^{3}+x^{2}-x-1 \text { and } x^{4}-x^{2}+1 \text { for } \hat{\theta}_{2}^{\prime} .
$$

These numbers are ordered as follows:

$$
\phi_{1}=\psi_{1}<\phi_{2}<\psi_{2}<\phi_{3}<\hat{\theta}_{2}^{\prime}<\psi_{3}<\phi_{4}<\psi_{4} \ldots
$$


Numerical values for the numbers in (2.1) to 3 decimal places are

$$
1.618<1.755<1.839<1.867<1.905<1.928<1.933<1.966 \text {. }
$$

The regular Pisot numbers associated with these limit points thus give an infinite number of infinite sequences of known Pisot numbers in [1,2]. Talmoudi [25, 26] showed that, for any $\delta>0$, apart from these regular Pisot numbers, there are only a finite number of Pisot numbers in $[1,2-\delta]$; we call these numbers irregular Pisot numbers. This result was made effective in [10], which gives an algorithm for determining the irregular Pisot numbers in an interval containing a limit point. The main difficulties occur for $\phi_{r}$ for $r \geq 3$. These have width exceeding $(\sqrt{5}+1) / 2$ while all of the other limit points have width 1 and were treated in the earlier paper [7]. The width $w(\beta)$ of a limit point $\beta$, which is defined in [7], gives a measure of the complexity of the tree which one must search to find all Pisot numbers in the neighborhood of $\beta$. There is a quantitative change in the complexity of the algorithm when $w(\beta)>(\sqrt{5}+1) / 2$, a case which is explored more fully in [10]. The paper [11] gives the details of the algorithm of [10] for the limit point $\phi_{3}=1.86676 \ldots$ (In those papers, $\phi_{r}$ and $\psi_{r}$ were denoted $\alpha_{r}$ and $\beta_{r}$, a notation we have temporarily abandoned because of the possibility of confusion with the many occurrences of $\beta$ in this paper).

\section{The Beta-EXPANSION FOR REgular Pisot Numbers}

The canonical beta-expansion of 1 to base $\beta>1$ is defined by the "greedy" algorithm: $\alpha_{0}=1, c_{n}=\left\lfloor\beta \alpha_{n-1}\right\rfloor$ and $\alpha_{n}=\beta \alpha_{n-1}-c_{n}$ for $n \geq 1$. Then $\alpha_{n}=$ $T^{n}(1)$, and

$$
1=\sum_{n=1}^{\infty} c_{n} \beta^{-n},
$$

where $0 \leq c_{n}<\beta$ for all $n$. The sum (3.1) is the beta-expansion of 1 to base $\beta$. We will often refer to the sequence $\left\{c_{n}\right\}$ as the beta-expansion for $\beta$. If this expansion is periodic, with preperiod length $m$ and period length $p$, we denote it by $c_{1} \ldots c_{p}$, if $m=0$, and by $c_{1} \ldots c_{m}: c_{m+1} \ldots c_{m+p}$ if $m>0$. For $\beta<2$, we can regard the expansion as a finite or infinite word in the alphabet $\{0,1\}$ and will use the notation of formal languages for these words where convenient. The word $c_{1} \ldots c_{m}$ is called the preperiod and $c_{m+1} \ldots c_{m+p}$ the period of the expansion. The exact conditions on $\left\{c_{n}\right\}$ for it to be the beta-expansion of 1 for some $\beta>1$ were determined by Parry [21, p. 407]: the sequence $c_{1}, c_{2}, \ldots$ must dominate, in the sense of lexicographic order, the shifted sequences $c_{k}, c_{k+1}, \ldots$, for all $k \geq 1$.

In computing the beta-expansion for an algebraic integer $\beta$ of degree $d$, the successive iterates $\alpha_{n}=T^{n}(1)$ lie in the ring $\mathbb{Z}[\beta]$ and hence can be represented exactly as $d$-vectors of integers, so the computation of $\alpha_{n}$ from $\alpha_{n-1}$ and $c_{n}$ uses only integer arithmetic. The only part of the algorithm that is best done in noninteger arithmetic is the computation of $c_{n}=\left\lfloor\beta \alpha_{n-1}\right\rfloor$. This must occasionally be done to fairly high accuracy if $\alpha_{n}$ is very small. The use of 50-digit precision for this step sufficed for all expansions described here. Even this step can be done with integer arithmetic by the use of resultants, but the extra time required does not make such an approach competitive with the use of floating-point arithmetic. 
All the computations described in this paper were done using the Maple computer algebra system [14]. The algorithm described in [7] was recoded using the arbitrary precision integer routines of Maple. The test for cyclotomic $Q(x)$ used the rootsquaring method described in [8] (and apparently rediscovered in [13]).

We begin by studying the beta-expansions for the regular Pisot numbers associated with the eight limit points listed in (2.1). There are either four or six families associated with each limit point, corresponding to the two or three choices for $A(x)$ and the two choices of sign in $x^{n} C(x) \pm A(x)$. In any given case, it is easy to observe the pattern in the expansions as a function of $n$ and to predict the expansion for general $n$. The three-step method of [12] can then be applied to prove the general result (see part (2) of the proofs of Propositions 5.1 and 5.2 below). We illustrate this below with some arbitrarily chosen examples. The conclusion from this is that for the regular Pisot numbers associated with the limit points $\phi_{r}$ and $\psi_{r}$ for $r \leq 4$, the factor $Q(x)$ is cyclotomic. We conjecture that this is the case for all $r$.

However, for the special limit point $\hat{\theta}_{2}^{\prime}$, with $C(x)=x^{4}-x^{3}-2 x^{2}+1$, if $A(x)=x^{4}-x^{2}+1$, then $x^{n} C(x)+A(x)$ has $Q(x)$ nonreciprocal if $n \geq 4$ is even, and $x^{n} C(x)-A(x)$ has $Q(x)$ nonreciprocal if $n \geq 7$ is odd, and also for $n=2$ and 3 (in these latter two cases, the corresponding root satisfies $\beta>2$ ). For $B(x)=x^{3}+x^{2}-x-1, x^{n} C(x) \pm B(x)$ has $Q(x)$ cyclotomic except in two cases: $x^{n} C(x)-B(x)$ with $n=2$ or 3 has $Q(x)$ nonreciprocal (again with $\beta>2$ ). More details of the expansions are given below.

We should first point out that the beta-expansions for the limit points $\phi_{r}, \psi_{r}$ and $\hat{\theta}_{2}^{\prime}$ are not complicated. The number $\phi_{r}$ is a simple beta-number with $p=2 r$, expansion $1^{r} 0^{r-1} 1$ and with complementary factor $Q(x)=\left(x^{r}-1\right) /(x-1)$. The number $\psi_{r}$ is a simple beta-number with $p=r+1$, expansion $1^{r+1}$ and $Q(x)=1$. Only $\hat{\theta}_{2}^{\prime}$ is not a simple beta-number; it has $(m, p)=(2,2)$, with expansion 11:01 = $11(01)^{\omega}$ and $Q(x)=1$.

For example, consider the regular Pisot numbers $\beta_{n}$ associated with $\psi_{2}$ which satisfy $P_{n}\left(\beta_{n}\right)=0$, where $P_{n}(x)=x^{n}\left(x^{3}-x^{2}-x-1\right)+\left(x^{3}-1\right)$. If $n$ is even, $P_{n}(x)$ is the minimal polynomial of $\beta_{n}$ while if $n$ is odd the minimal polynomial is $P_{n}(x) /(x+1)$. We find that $\beta_{n}$ is a simple beta-number with beta-expansion given, for $n \geq 2$, by

$$
\begin{array}{rll}
(110)^{k} 0110^{3 k} 1 & \text { if } & n=3 k+1 \\
(110)^{k} 1010^{3 k+1} 1 & \text { if } & n=3 k+2, \\
(110)^{k+1} 0^{3 k+2} 1 & \text { if } & n=3 k+3 .
\end{array}
$$

Note that the expansions in (3.2) converge to $(110)^{\omega}$ as $n \rightarrow \infty$. This is a legitimate expansion for $\psi_{2}$ but not the canonical expansion. The corresponding $Q(x)$ in these three cases is $Q_{n}(x)=x^{3 k}+x^{3 k-3}+\cdots+x^{3}+1$ if $n$ is even or $Q_{n}(x)(x+1)$ if $n$ is odd. As in [12], all that is needed here is to verify from Parry's [21] criterion that the expansions $c_{1} \ldots c_{m}$ given in (3.2) are the beta-expansions of 1 for some $\beta>1$ and then to verify that $P_{n}(x) Q_{n}(x)=x^{m}-c_{1} x^{m-1}-\cdots-c_{m}=R_{n}(x)$. This is an elementary calculation. The case $n=1$ follows a different pattern but gives a simple beta-number with cyclotomic $Q$.

The numbers in the previous paragraph are to the left of $\psi_{2}$. Taking the other sign, we obtain $\beta_{n}>\psi_{2}$ satisfying $P_{n}(x)=x^{n}\left(x^{3}-x^{2}-x-1\right)-\left(x^{3}-1\right)$. Here, $P(x)=P_{n}(x)$ if $n$ is odd, and $P_{n}(x) /(x+1)$ if $n$ is even. For $n \geq 3$, the expansion 
of $\beta_{n}$ is periodic with $(m, p)=(3, n)$ and is given explicitly by $111: 0^{n-3} 110$. Here the expansions converge to 111, the canonical expansion of $\psi_{2}$, as $n \rightarrow \infty$. The complementary factor is 1 if $n$ is odd and is $x+1$ if $n$ is even. The two cases $n=1$ and 2 have $\beta_{n}>2$ and periodic expansions 2:101 and 2:001, respectively, both with $Q(x)=1$.

For the expansions for regular Pisot numbers associated with $\psi_{r-1}$ satisfying $P_{n}(x)=x^{n}\left(x^{r}-x^{r-1}-\cdots-x-1\right)+\left(x^{r}-1\right)$, it seems that $Q$ is the product of $x^{r k}+x^{r(k-1)}+\cdots+x^{r}+1$ times the cyclotomic factor of $P_{n}$, for sufficiently large $n$, but this has only been verified for $r \leq 4$. Taking the same definition but with the \pm sign chosen to be - , one finds that the expansion is periodic with $(m, p)=(r, n)$, or explicitly $1^{r}: 0^{n-r} 1^{r-1} 0$, and that $Q$ is the cyclotomic factor of $P_{n}$. Again, this has been verified only for $r \leq 4$.

The expansions for regular Pisot numbers corresponding to the limit points $\phi_{r}$ tend to follow more complicated patterns than for those associated to the $\psi_{r}$, presumably because the beta-expansion of $\phi_{r}$ is somewhat more complicated than that of $\psi_{r}$. For example, associated with $\phi_{3}$, we have $\beta_{n}$ with minimal polynomial

$$
P(x)=\left(x^{n}\left(x^{4}-2 x^{3}+x-1\right)+\left(x^{3}-x+1\right)\right) / G_{n}(x),
$$

where $G_{n}(x)$ is the cyclotomic factor of the numerator. We find that $\beta_{n}$ is a simple beta-number for all $n$ with $p$ depending on $n \bmod 6$ in the following way:

$$
p= \begin{cases}2 n+3 & \text { if } n \equiv 0 \bmod 6 \\ n & \text { if } n \equiv 1 \bmod 6 \\ 4 n-1 & \text { if } n \equiv 2 \bmod 6 \\ 2 n & \text { if } n \equiv 3 \bmod 6 \\ 2 n-1 & \text { if } n \equiv 4 \bmod 6 \\ 2 n-2 & \text { if } n \equiv 5 \bmod 6\end{cases}
$$

For example, the explicit expansion for $n=6 k+2$ is $1^{3} 0^{3}(10)^{3 k+1} 01(10)^{3 k} 0^{2} 1^{3} 0^{3} 1$. Note the convergence to the noncanonical expansion $1^{3} 0^{3}(10)^{\omega}$ for $\phi_{3}$. The factor $Q$ in this case is a cyclotomic polynomial of degree $3 n-4$.

The sort of periodic behavior exhibited in (3.3) was found for all of the 36 families of regular Pisot numbers we considered. For $\phi_{r}$ the modulus is a factor of $2 r$ and for $\psi_{r}$ the modulus is a factor of $r+1$, at least for $r \leq 4$. Note that these are the length of the beta-expansions for $\phi_{r}$ and $\psi_{r}$. Although these regularities have as yet no satisfactory explanation, they lend credibility to the conjectures concerning $\phi_{r}$ and $\psi_{r}$ for $r>4$.

As mentioned earlier, the only noncyclotomic $Q$ found among the regular Pisot numbers in $[1,2]$ correspond to the exceptional limit point $\hat{\theta}_{2}^{\prime}=1.90516 \ldots$, which has minimal polynomial $C(x)=x^{4}-x^{3}-2 x^{2}+1$. Let $A(x)=x^{4}-x^{2}+1$ and $B(x)=x^{3}+x^{2}-x-1$. Notice that $C(x) C(1 / x)=A(x) A(1 / x)+B(x) B(1 / x)$, so that, for example, the cyclotomic factor of $P_{n}(x)=x^{n} C(x) \pm A(x)$ is $G_{n}(x)=$ $\operatorname{gcd}\left(P_{n}(x), B\right)$.

Consider first $x^{n} C(x)+A(x)$. For odd $n, Q(x)$ is cyclotomic. More precisely, if $n=1, \beta_{1}=1.32471 \ldots$ is the smallest Pisot number with minimal polynomial $x^{3}-x-1$; it is simple with $p=5$, expansion 10001 , and $Q(x)=x^{2}-x+1$. if $n \geq 3$ is odd, then $\beta_{n}$ is a simple beta-number with $p=n+2$ and $Q(x)=1$. 
Continuing with $x^{n} C(x)+A(x)$, if $n$ is even, we find that $Q(x)$ is cyclotomic for $n=2$ and noncyclotomic for $n \geq 4$. For $n=4, \beta_{4}=1.80421 \ldots$ has minimal polynomial

$$
P(x)=x^{7}-2 x^{5}-2 x^{4}-x-1,
$$

and we find that $(m, p)=(6,14)$ and that $Q(x)$ is a nonreciprocal polynomial of degree 13, namely

$$
Q(x)=x^{13}-x^{12}+x^{11}-x^{9}+2 x^{8}-2 x^{7}+2 x^{6}-x^{5}+x^{3}-x^{2}+x-1 .
$$

We will see in the next section that this $\beta_{4}$ is the unique Pisot number in $[1,2]$ of degree at most 7 for which $Q(x)$ is noncyclotomic. If $n \geq 6$ is even, then $P_{n}(x)=x^{n} C(x)+A(x)$ has $(m, p)=(n+2,2 n+4)$ and $Q$ is a nonreciprocal polynomial of degree $2 n+3$.

The polynomial $Q(x)$ of (3.5) occurs again in Proposition 5.5, where we show that $Q\left(x^{k}\right)$ occurs as a complementary factor for any $k \geq 1$ for a certain $\beta_{k}$ of degree $6 k+1$.

For $x^{n} C(x)-A(x), Q(x)$ is nonreciprocal when $n=2$ and 3 , both with $\beta>2$, and when $n$ is odd and $n \geq 7$; otherwise, $Q(x)$ is cyclotomic. In detail, $\beta_{1}=2.58808 \ldots$ has $P(x)=x^{5}-2 x^{4}-2 x^{3}+x^{2}+x-1,(m, p)=(3,2)$ and $Q=1$. For $n=2$, $\beta_{2}=2.19659 \ldots$ has

$$
P(x)=x^{5}-2 x^{4}-x^{3}+x^{2}+x-1,
$$

$(m, p)=(3,5)$ and

$$
Q(x)=x^{3}+x+1
$$

nonreciprocal; the explicit beta-expansion here is 200: 20001. This is the simplest example of a noncyclotomic $Q$ found among the regular Pisot numbers, but note that it occurs for $\beta>2$. Similarly, $\beta_{3}=2.05295 \ldots$ has

$$
P(x)=x^{7}-x^{6}-2 x^{5}-x^{4}+x^{3}+x^{2}-1,
$$

$(m, p)=(4,12)$ and the nonreciprocal

$$
Q(x)=x^{9}-x^{8}+x^{7}+x^{4}+x^{2}+1=(x+1)\left(x^{2}+1\right)\left(x^{2}-x+1\right)\left(x^{4}-x^{3}+1\right) .
$$

For $n \geq 4, x^{n} C(x)-A(x)$ has $\beta_{n}<2$ and if $n$ is even, then $\beta_{n}$ is a simple beta-number with $p=12$ for $n=4$ and $p=2 n$ for $n \geq 6$; the corresponding $Q$ is the cyclotomic polynomial $\left(x^{n-2}-1\right) /(x-1)$.

For $n$ odd, $\beta_{5}$ is also a simple beta-number with a cyclotomic $Q$, but for $n \geq 7$, $(m, p)=(2 n, n)$ with a nonreciprocal $Q(x)=\left(x^{2 n-2}-x^{n-1}-x^{n-3}+1\right) /\left(x^{2}-1\right)$. For these $n$, the beta-expansion for $\beta_{n}$ is

$$
111(01)^{(n-5) / 2} 10100(01)^{(n-7) / 2} 0011: 10^{n-3} 10 .
$$

For $x^{n} C(x)+\epsilon B(x), \epsilon= \pm 1, Q(x)$ is cyclotomic except in two cases, both with $\epsilon=-1$ and having $\beta>2$, namely $\beta_{2}=2.10213 \ldots$, which has $(m, p)=(11,2)$ and $Q(x)=x^{7}-x^{6}+x^{4}-x+1$, nonreciprocal, and $\beta_{3}=2.01043 \ldots$ with a nonreciprocal $Q$ of degree 30 which has a cyclotomic factor $\left(x^{13}-1\right) /(x-1)$.

So, the regular Pisot numbers associated with $\hat{\theta}_{2}^{\prime}$ already provide answers to the questions posed by Katai and Frougny. The examples (3.4) and (3.6) are reasonably simple, although one might hope for an example with $\beta<2$ for which $Q(x)$ has smaller degree. The regular Pisot numbers in $[1,2]$ also apparently provide no examples in which $Q(x)$ is a noncyclotomic reciprocal polynomial. For these, at least for $1<\beta<2$, it seems that we must look at the irregular Pisot numbers. 


\section{The Beta-expansions for irRegular Pisot numbers}

For any $\delta>0$, the set of irregular Pisot numbers in $[1,2-\delta]$ is finite. It can be enumerated by using the algorithms of [7] and [10]. The computation in the neighborhood of the limit points $\phi_{r}$, for $r \geq 3$, requires some preliminary analysis which has only been completely carried out in the case of $\phi_{3}=1.86676 \ldots$ [11], so here we carried out a complete enumeration only for the intervals $[1,1.9324]$ and [1.9333, 1.96].

We will find it convenient to use the abbreviations $\mathrm{C}$ for "cyclotomic", NR for "nonreciprocal" and NC for "reciprocal but noncyclotomic". In addition, we will say that $\beta$ is of type $\mathrm{C}, \mathrm{NR}$ or NC according to whether the complementary factor $Q$ is $\mathrm{C}, \mathrm{NR}$ or NC.

The algorithm of [7] is also easily adapted to enumerate all elements of $S \cap[a, b]$ of degree at most $d$, where $[a, b]$ is any finite interval. This set is clearly finite since it is a set of algebraic integers of bounded degree with all conjugates bounded, so it is obvious that it can be enumerated by the naive approach of bounding the coefficients of the polynomials involved, using elementary symmetric functions. However, the method of [7], which uses Schur's algorithm in the manner pioneered by Dufresnoy and Pisot [15], searches through a much smaller set. We used this to enumerate $S \cap[1.9,2]$ up to degree $50, S \cap[1.96,2]$ up to degree 60 , and $S \cap[2,2.2]$ up to degree 20 .

4.1. The interval $[1,2]$. For example, enumerating $S \cap[1,1.76]$ (which includes the two smallest limit points), we find only points of type C. But, in $S \cap[1.76,1.78]$ there are 3 examples of type NR and 1 of type NC. We thus have the smallest Pisot number of type NR: $\beta=1.76478 \ldots$, of degree 16 , with minimal polynomial

$$
P=[1,0,-1,-1,-2,-3,-2,-2,-2,0,1,1,2,2,1,1,1]=x^{16}-x^{14}-\ldots
$$

for which $(m, p)=(113,51)$. (We will use the notation $\left[a_{0}, a_{1}, \ldots, a_{d}\right]$ for the polynomial $a_{0} x^{d}+\cdots+a_{d}$, whenever convenient). We omit the beta-expansion and $Q$ for reasons of space ( $Q$ is a nonsparse polynomial of degree 148).

The second-smallest $\beta$ of type NR is $\beta=1.76489 \ldots$, of degree 11 , with minimal polynomial

$$
[1,0,-2,-2,-1,0,2,2,1,0,-1,-1],
$$

which has $(m, p)=(30,5)$ and $Q(x)=\left(x^{2}-x+1\right)\left(x^{22}+x^{15}+x^{8}-x^{7}-1\right)$.

The smallest $\beta$ of type NC is $\beta=1.76789 \ldots$, of degree 12 , with minimal polynomial

$$
P=[1,0,-1,-2,-2,-1,-1,-1,-1,0,1,1,1] .
$$

It has $(m, p)=(4,34)$ and

$$
Q(x)=F_{4}(x) F_{6}(x) F_{12}(x) F_{30}(x) L(-x)
$$

where $F_{q}(x)$ is the minimal polynomial of the $q$ th roots of unity and

$$
L(x)=x^{10}+x^{9}-x^{7}-x^{6}-x^{5}-x^{4}-x^{3}+x+1
$$


is the minimal polynomial of Lehmer's number, the smallest known Salem number. The polynomial $L(x)$ occurs elsewhere: e.g. there is a simple beta-number $\beta=$ $1.93723 \ldots$, of degree 16 , with minimal polynomial

$$
P=[1,-2,1,-1,-1,0,-1,0,-1,0,-1,0,0,-1,0,0,-1]
$$

for which $Q(x)=\left(x^{2}+x+1\right) L\left(-x^{2}\right)$.

The next interval considered, $[1.78,1.8]$, also contained 3 numbers of type NR and one of type NC. The latter is $\beta=1.79002 \ldots$ with minimal polynomial of degree 15 ,

$$
P=[1,0,-1,-2,-2,-2,-1,-1,0,1,1,1,1,1,1,1]
$$

which has $(m, p)=(26,7)$ and a noncyclotomic irreducible $Q$ of degree 18 , namely

$$
Q=[1,-1,0,1,-1,1,-1,0,1,-1,1,0,-1,1,-1,1,0,-1,1],
$$

having largest root of absolute value $1.12661 \ldots$ In $[1,2]$ we found only one other $\beta$ of type NC with $\operatorname{deg}(Q)=18$ and none with $\operatorname{deg}(Q)$ smaller than 18 .

One of the other Pisot numbers in this interval for which $Q$ is fairly simple is $\beta=1.79130 \ldots$ with $P(x)$ of degree 18 given by

$$
P=[1,-2,1,-1,0,0,-1,1,-1,0,1,-1,1,-1,0,0,-1,1,-1] .
$$

This has $(m, p)=(16,15)$ and $Q(x)=(x+1)\left(x^{12}+x^{3}-1\right)$. This example is generalized in Proposition 5.4.

The simplest nonreciprocal polynomials are trinomials. Proposition 5.4 shows that $Q(x)=x^{4 k}+x^{k}-1$ occurs for $\beta<2$ for every even $k \geq 4$, giving for example $Q(x)=x^{16}+x^{4}-1$. Among the "sporadic" examples, we find $Q(x)=x^{16}+x^{5}-1$, which occurs for $\beta=1.95114 \ldots$, of degree 23 , with

$$
P=[1,-1,-1,-1,-1,0,-1,0,-1,0,1,0,1,0,1,1,0,0,-1,0,0,0,0,-1] .
$$

with $(m, p)=(31,8)$.

Enumerating the subset of $S \cap[1,2]$ of degree at most 7 , we find that there are 71 such numbers, only one of which is not of type C. This is $\beta=1.80421 \ldots$, with the minimal polynomial $P(x)$ given in $(3.4),(m, p)=(6,14)$ and $Q(x)$ the nonreciprocal polynomial of degree 13 given in (3.5). We have already identified this $\beta$ as a regular Pisot number associated with $\hat{\theta}_{2}^{\prime}$. Thus, the smallest degree of $P(x)$ with $\beta<2$ for which $Q(x)$ is noncyclotomic is 7 and the $P(x)$ of (3.4) is the unique example of this degree.

Considering next the possible degrees of the complementary factor $Q(x)$ for $\beta<2$, we find that the noncyclotomic complementary factor $Q$ of smallest degree for $\beta<2$ is

$$
Q(x)=x^{6}+x^{5}-x^{3}-x^{2}+1,
$$

which occurs for a $\beta=1.85896 \ldots$, of degree 15 , with

$$
P=[1,-2,1,-1,0,-1,0,-1,0,0,0,0,0,1,-1,1],
$$


and $(m, p)=(14,7)$. The expansion here is 11100010100001:0100010. Since a lower-degree noncyclotomic complementary factor might be associated with a $P(x)$ of larger degree than we have considered, we cannot claim that this is the smallest degree of such $Q(x)$ possible for $\beta<2$, only that it is the smallest degree we were able to find.

Clearly related to the $\beta$ of the previous paragraph is $\beta=1.85930 \ldots$, of degree 18 , with

$$
P=[1,-2,1,-1,-1,1,-1,0,1,-1,1,0,-1,1,-1,0,1,-1,1],
$$

which has $(m, p)=(21,7), Q(x)=\left(x^{6}+x^{5}-x^{3}-x^{2}+1\right)\left(x^{4}+1\right)$ and expansion 111000101000110100001: 0100010. Note that the periodic part of the expansions are identical and the preperiod of the second differs from the first only by the insertion of the sequence 0001101.

A pattern of coefficients which seems to occur regularly in the minimal polynomials for $\beta$ of type NC is exemplified by the following few examples:

$$
P=[1,0,-1,-2,-3,-4,-4,-4,-3,-2,-1,0,0,1,1,1,1,1,1,1,1,1,1,1,1],
$$

of degree 24 , with $\beta=1.95656 \ldots,(m, p)=(16,235)$ and $\operatorname{deg}(Q)=227$,

$$
P=[1,0,-1,-2,-3,-4,-4,-4,-3,-2,-1,-1,0,1,1,1,1,1,1,1,1,1,1,1],
$$

of degree 23 , with $\beta=1.95679,(m, p)=(15,69)$ and $\operatorname{deg}(Q)=61$, and

$$
P=[1,0,-1,-2,-3,-4,-3,-3,-2,-1,0,0,1,1,1,1,1],
$$

of degree 16 , with $\beta=1.93680 \ldots,(m, p)=(8,61)$ and $\operatorname{deg}(Q)=53$. See also $(4.2)$ and (4.3). There are also many having similar patterns which are NR rather than NC, e.g. (4.1).

The results of an enumeration of $S \cap[1.9,2]$ up to degree 50 are summarized in Table 1 , where we classify the $\beta \in S \cap[a, b]$ of degree at most $d$ according to their type (C, NR or NC). Among these numbers, there is a $\beta$ of type $\mathrm{C}$, of degree 50 with $\operatorname{deg}(Q)=624$, one of type NR, of degree 50 with $\operatorname{deg}(Q)=3708$, and one of type NC of degree 28 with $\operatorname{deg}(Q)=992$.

Table 1 also contains a summary of an enumeration of $S \cap[1.96,2]$ up to degree 60. Among these is a $\beta$ of type NR of degree 53 with $\operatorname{deg}(Q)=13551$. It is interesting to observe that the largest value of $\operatorname{deg}(P)$ found for $\beta<2$ of type NC is 36 . That is, there are no numbers with $\beta<2$ of type NC with degrees between 37 and 60 . This suggests that perhaps the set of Pisot numbers of type NC in $[1,2]$ is finite. Note that Proposition 5.2 of the next section shows that the set of Pisot numbers of type $\mathrm{NC}$ in $[2,2+\delta]$ is infinite for any $\delta>0$.

TABLE 1

\begin{tabular}{cccccc} 
interval & $\mathrm{d}$ & total & $\mathrm{C}$ & $\mathrm{NR}$ & $\mathrm{NC}$ \\
\hline$[1,1.9]$ & 50 & 1234 & 1173 & 54 & 7 \\
{$[1.9,2]$} & 50 & 7012 & 6820 & 161 & 31 \\
{$[1.96,2]$} & 60 & 8437 & 8344 & 77 & 16 \\
{$[2,2.1]$} & 20 & 3002 & 1429 & 1310 & 263 \\
{$[2.1,2.2]$} & 20 & 7008 & 2933 & 3884 & 191
\end{tabular}


For type C, the largest value of $\operatorname{deg}(Q)$ observed in this enumeration is 896 , attained for a $\beta$ of degree 60 . Indeed, an examination of the data suggests a sequence of regular Pisot numbers of type $\mathrm{C}$ of degree $2 k+3$ for which $\operatorname{deg}(Q) \sim k^{2}$. Specifically, let

$$
P_{k}(x)=x^{k+2}\left(x^{k}(x-2)+x-1\right)-\left(x^{k}-x+1\right) .
$$

Then $P_{k}(x)$ is the minimal polynomial of a regular Pisot number $\beta_{k}$ associated with the limit point $\phi_{k}$. This is a "diagonal" sequence for which $\beta_{k} \rightarrow 2$ as $k \rightarrow \infty$. We can prove, by a technique analogous to the proofs of Propositions 5.1 and 5.2 below, that $\beta_{k}$ is a simple beta-number with $p=p_{k}$, where $p_{k}=k^{2}+O(k)$. More precisely, if $Q_{k}$ denotes the complementary factor, then $\operatorname{deg}\left(Q_{k}\right)=p_{k}-(2 k+3)$ is given by

$$
\operatorname{deg}\left(Q_{k}\right)=k(k-1)+\left(\frac{3}{2}+\frac{1}{2}(-1)^{k+1}\right)(k-1)+(-1)^{k+1}-1 .
$$

Thus, $\operatorname{deg}\left(Q_{k}\right) \sim \frac{1}{4}\left(\operatorname{deg}\left(P_{k}\right)\right)^{2}$, which seems to be the maximal rate of growth of $\operatorname{deg}(Q)$ relative to $\operatorname{deg}(P)$ for numbers of type $C$ in $[1,2]$.

We will show in the next section that there are infinitely many numbers of type NR in $[1,2]$ in addition to the two families of regular Pisot numbers which accumulate at $\hat{\theta}_{2}^{\prime}$. These examples accumulate at 2 , which is consistent with Talmoudi's theorem about regular Pisot numbers and our conjecture that the regular Pisot numbers corresponding to the limit points $\phi_{r}$ and $\psi_{r}$ are all of type $\mathrm{C}$. The three sequences of irregular Pisot numbers described in Propositions 5.3-5.5 were found by examining the numbers of type NR in $S \cap[1.96,2]$ for which $\operatorname{deg}(Q)$ is relatively small.

4.2. The interval $[2,2.2]$. Once one begins to consider $\beta>2$, it appears that noncyclotomic $Q$ are quite common. Some data to illustrate this are found in Table 1. For $\beta<2$, notice that less than $5 \%$ of the $Q$ are noncyclotomic (NR or NC), but for $\beta>2$, more than $55 \%$ are noncyclotomic. So, for $\beta>2$, we will tend to be impressed only by such $Q$ of very large or very small degree. On the other hand, beta of type $\mathrm{NC}$ are still not very common when $\beta>2$.

Recall that the smallest degree of noncyclotomic $Q$ found for $\beta<2$ was 6 , and that, for $\beta>2$, we have already found one of degree 3 , namely $Q(x)=x^{3}+x+1$ for the $P(x)$ of (3.6) associated with $\hat{\theta}_{2}^{\prime}$. In our search up to degree 20 of $[2,2.2]$, we did not find this complementary polynomial again, but found 30 occurrences of $Q(x)=x^{3}-x^{2}+1$. The simplest occurred for the $\beta=2.09430 \ldots$ of degree 5 with $P=[1,-1,-1,-2,-1,-1]$, a simple beta-number with expansion 20001111. This $P$ is the case $k=1$ of the polynomials considered in Proposition 5.1.

Interestingly, 26 of the 30 numbers with $Q=x^{3}-x^{2}+1$ occur in a small neighborhood of $2.094 \ldots$ and are clearly related to this last example. For example, there is a degree- $15 \beta=2.09485 \ldots$ with

$$
P=[1,-1,-1,-2,-1,-1,0,0,0,0,-1,0,0,1,1,1],
$$

having $(m, p)=(8,10)$ and expansion 20001111:0010001100, and a degree-13 $\beta=$ $2.09669 \ldots$ with

$$
P=[1,-1,-1,-2,-1,-1,0,0,-1,0,0,1,1,1],
$$


having $(m, p)=(8,8)$ and expansion 20001111: 10001100, both with $Q=x^{3}-x^{2}+1$.

The numbers just described are all of type NR. For type NC, recall that the smallest value of $\operatorname{deg}(Q)$ found for $[1,2]$ was 18 . For $[2,2.2]$, the smallest value of $\operatorname{deg}(Q)$ found for type NC was 10 . One of these is the case $k=4$ of Proposition 5.2 .

In the other direction, there are 16 numbers in $[2,2.1]$ for which $\operatorname{deg}(Q) \geq 1000$, all of type NR, the largest value of $\operatorname{deg}(Q)$ being 5876 . In this interval there are 27 of type NC with $\operatorname{deg}(Q) \geq 100$, the largest value of $\operatorname{deg}(Q)$ being 319 . There are none of type $\mathrm{C}$ with $\operatorname{deg}(Q) \geq 100$.

In the interval $[2.1,2.2]$, there are $164 \beta$ for which $\operatorname{deg}(Q) \geq 1000$, all of type $\mathrm{NR}$, the largest value of $\operatorname{deg}(Q)$ being 8661 . There are just 4 of type NC with $\operatorname{deg}(Q) \geq 100$ and only two of type $\mathrm{C}$ with $\operatorname{deg}(Q) \geq 100$.

It is interesting to note that the ratio of $\#(\mathrm{NC})$ to $\#(\mathrm{NR})$ is much larger in $[2,2.1]$ than in $[2.1,2.2]$. The reason for this is unknown.

\section{Some infinite families of Pisot numbers}

Extrapolating from patterns observed in the data described above, we find the following interesting families of Pisot numbers. The first two have $\beta_{k}>2$ and the next three have $\beta_{k}<2$. In all cases, it is easy to see that $\beta_{k} \rightarrow 2$ as $k \rightarrow \infty$.

Proposition 5.1. For each $k \geq 1$, let

$$
P_{k}=x^{2 k+1}(x-2)-x^{k}(x-1)+1 .
$$

Then $P_{k}(x) /(x-1)$ is the minimal polynomial of a Pisot number $\beta_{k}>2$ with simple beta-expansion $20^{k+1} 1^{2 k}$ having $p=3 k+2$ and

$$
Q_{k}=x^{k+1}-x^{k}+1 \text {. }
$$

Proposition 5.2. For each $k \geq 2$, let

$$
P_{k}=x^{2 k}(x-2)-x^{k-1}(x-1)+1,
$$

and

$$
Q_{k}=x^{2 k+2}-x^{2 k+1}+x^{k+1}-x+1,
$$

which is reciprocal but noncyclotomic for sufficiently large $k$. Then $P_{k}(x) /(x-1)$ or $P_{k}(x) /\left(x^{2}-1\right)$, depending on whether $k$ is even or odd, is the minimal polynomial of a Pisot number $\beta_{k}>2$ with beta-expansion $20^{k}: 01^{k-1} 01^{k-2} 0110^{k-2} 1$ having $(m, p)=(k+1,3 k+1)$ and complementary factor $Q_{k}(x)$ or $Q_{k}(x)(x+1)$, for $k$ even or odd, respectively.

Proposition 5.3. For each $k \geq 4$, let

$$
\begin{aligned}
P_{k}=x^{5 k+1}(x-2) & +x^{4 k}(x-1)-x^{3 k+1}(x-1) \\
& -x^{2 k}(x-1)+x^{k+1}(x-1)+(x-1) .
\end{aligned}
$$

Then $P_{k}=S_{k} T_{k}$, where $S_{k}(x)$ is the minimal polynomial of a Pisot number $\beta_{k}<2$ and $T_{k}(x)$ is cyclotomic, being either 1 if $k \not \equiv 3 \bmod 10$, or $x^{4}-x^{3}+x^{2}-x+1$ if $k \equiv 3 \bmod 10$. The beta-expansion of $\beta_{k}$ has $(m, p)=(6 k+2,3 k+1)$ and complementary factor $Q_{k}(x) T_{k}(x) /(x-1)$, where

$$
Q_{k}=x^{4 k+2}-x^{3 k+1}+x^{2 k}-x^{k+1}-x^{2}+1 .
$$


Proposition 5.4. For each $k \geq 3$, let

$$
\begin{aligned}
P_{k}=x^{6 k+1} & (x-2)+x^{5 k}\left(x^{2}-x+1\right)-x^{4 k+1}(x-1) \\
& -x^{3 k}\left(x^{2}-2 x+1\right)-x^{2 k}+x^{k}\left(x^{2}-x+1\right)-x+1,
\end{aligned}
$$

and let

$$
Q_{k}=x^{4 k}+x^{k}-1
$$

Then $P_{k}(x) /(x-1)$ or $P_{k}(x) /\left(x^{2}-1\right)$, depending on whether $k$ is even or odd, is the minimal polynomial of a Pisot number $\beta_{k}<2$ with $(m, p)=(5 k+1,5 k)$ and complementary factor $Q_{k}(x)$ or $(x+1) Q_{k}(x)$, for $k$ even or odd, respectively.

Proposition 5.5. For each $k \geq 1$, let

$$
\begin{aligned}
P_{k}=x^{8 k-1}(x-2) & +x^{7 k-1}(x-2)+x^{6 k-1}-x^{5 k-1}(x-3) \\
- & x^{4 k-1}(x-1)-x^{3 k-1}(x+1)-x^{2 k-1}+x^{k}+1
\end{aligned}
$$

and let $Q_{k}(x)=Q\left(x^{k}\right)$, where $Q(x)$ is the polynomial of degree 13 of equation (3.5). Then $P_{k}(x) /(x-1)$ is the minimal polynomial of a Pisot number $\beta_{k}<2$ with $(m, p)=(7 k-1,14 k)$ and complementary factor $Q_{k}(x)$.

Remark 5.1. It is not obvious that any of the polynomials $P_{k}$ in Propositions 5.15.5 define Pisot numbers. This can be established in all cases by introducing a suitable homotopy parameter, a method used in [6] for a different purpose, as we illustrate below in the proof of Proposition 5.3. We present a different proof for the $P_{k}$ of Proposition 5.1, which illustrates a different use of a homotopy parameter. We can establish that the polynomial $P_{k}$ of Proposition 5.2 defines a Pisot number, using the known structure of the Pisot numbers as explained below, but it seems that the other four examples cannot be dealt with by this method.

Remark 5.2. Proposition 5.1 shows that the trinomials $Q_{k}(x)=x^{k+1}-x^{k}+1$, the "simplest" nonreciprocal polynomials, occur as the complementary factors of a fairly simple sequence of Pisot numbers $\beta_{k} \downarrow 2$. We do not have such a simple example for a sequence $\beta_{k} \uparrow 2$, and the evidence of our current studies suggests no such example exists. The sequence $P_{k}$ of Proposition 5.4 does have the simple complementary factor $x^{4 k}+x^{k}-1$, at least for $k \geq 3$ and even, but the polynomial $P_{k}$ is much more complicated.

Remark 5.3. The examples of Proposition 5.2 show there are infinitely many $\beta$ of type $\mathrm{NC}$ in any interval $[2,2+\delta]$. There are only 38 known numbers of type $\mathrm{NC}$ in $[1,2]$, the largest degree of such numbers being 36 , in spite of a search up to degree 60. As remarked above, this suggests that there may only be finitely many $\beta$ of type $\mathrm{NC}$ in $[1,2]$, but this has not been settled conclusively. Certainly, no simple example such as that of Proposition 5.2 seems possible with $\beta_{k} \uparrow 2$.

Proof of Proposition 5.1. (1) We begin by proving that $P_{k}(x) /(x-1)$ is a Pisot polynomial. For given $k$, let $t$ be real and consider the more general polynomial

$$
P(x, t)=x^{2 k+1}-\left(x^{2 k+1}-1\right) /(x-1)-t x^{k},
$$

so that $P_{k}(x) /(x-1)=P(x, 1)$. It is well known that $P(x, 0)$ is a Pisot polynomial, indeed it is the minimal polynomial of the limit point $\psi_{2 k+1}$ considered in $\S 2$. The 
zeros of $P(x, t)$ are continuous functions of $t$. Clearly, for $t \geq-1, P(x, t)$ has a unique positive root which is monotone increasing with $t$ and hence varies from $\psi_{2 k+1}$ to $\beta_{k}$ as $t$ increases from 0 to 1 . If $P(x, 1)$ is not a Pisot polynomial, then it must have some other roots outside or on the unit circle and hence, following the paths of the roots of $P(x, t)$ as $t$ varies from 0 to 1 which start at the $2 k$ roots of $P(x, 0)$ inside the unit circle, and end at the roots of $P(x, 1)$, one of these must cross the unit circle for some $0<t \leq 1$. That is, $P(x, t)$ must have a root on the unit circle for some $t$ with $0<t \leq 1$. We will show that this cannot occur by showing that $P(x, t)$ has no roots on the unit circle for $-2<t<2$.

The roots of $P(x, t)$ on the unit circle are also roots of the reciprocal polynomial

$$
P^{*}(x, t)=x^{2 k+1} P(1 / x, t)=1-x\left(x^{2 k+1}-1\right) /(x-1)-t x^{k+1} .
$$

The common roots of $P(x, t)$ and $P^{*}(x, t)$ are also roots of $x P(x, t)-P^{*}(x, t)=$ $x^{2 k+2}-1$, i.e., $(2 k+2)$ nd roots of 1 . We test whether these can be roots of $P(x, t)$ by substitution. First test $x=1$, and find $P(1, t)=-2 k-t$, which can vanish only if $t=2 k \geq 2$. Otherwise, let $\omega \neq 1$ satisfy $\omega^{2 k+2}=1$. Then

$$
\begin{aligned}
P(\omega, t) & =\omega^{-1}-\left(\omega^{-1}-1\right) /(\omega-1)-t \omega^{k} \\
& =\left(2 \omega^{-k-1}-t\right) \omega^{k}=( \pm 2-t) \omega^{k}
\end{aligned}
$$

which can vanish only if $t= \pm 2$. Hence $P(x, t)$ has no roots on the unit circle if $-2<t<2$ and thus must have the same numbers of roots inside or outside the unit circle as does $P(x, 0)$, i.e. 1 outside and $2 k$ inside. In particular, $P(x, 1)$ is a Pisot polynomial.

(2) The remaining part of the proof follows the pattern of [12]. We observe from Parry's criterion [21] that the finite sequence $20^{k+1} 1^{2 k}$ is indeed the canonical beta-expansion of 1 for some $\beta>1$ : because of the leading 2 , the word $20^{k+1} 1^{2 k} 0^{\omega}$ clearly dominates all of its left shifts. This $\beta$ has characteristic polynomial

$$
R_{k}=x^{3 k+2}-2 x^{3 k+1}-\left(x^{2 k}-1\right) /(x-1) .
$$

Now we simply check that

$$
\begin{aligned}
P_{k} Q_{k} & =\left\{x^{2 k+1}(x-2)-x^{k+1}+x^{k}+1\right\}\left\{x^{k}(x-1)+1\right\} \\
& =x^{3 k+1}(x-2)(x-1)-x^{2 k}+1=(x-1) R_{k} .
\end{aligned}
$$

The left member of (5.1) has a unique root $\beta_{k}>1$ while the right member has $\beta>1$ as a root. Thus $\beta=\beta_{k}$, so the beta expansion for $\beta_{k}$ must be as claimed, and the complementary factor is $Q_{k}$.

Proof of Proposition 5.2. (1) Again we first show that $P_{k}(x) /(x-1)$ or $P_{k}(x) /\left(x^{2}-1\right)$ is a Pisot polynomial according to whether $k$ is even or odd. A proof very similar to that in Proposition 5.1 is possible, and we leave this to the reader. Instead, we give a proof based on known facts from the theory of the Pisot numbers. First observe that we may write

$$
P_{k}=x^{k}\left(x^{k+1}-2 x^{k}-1\right)+\left(x^{k-1}+1\right) .
$$


For $|x|=1$, since $\bar{x}=1 / x$, we may verify the following polynomial identity (cf. [3, p. 142]):

$$
\left|x^{k+1}-2 x^{k}-1\right|^{2}=\left|\left(x^{k}+1\right)(x-1)\right|^{2}+\left|x^{k-1}+1\right|^{2} .
$$

The polynomial $x^{k+1}-2 x^{k}-1=x^{k}(x-2)-1$ is easily seen to be a Pisot polynomial by Rouché's theorem, since $|x-2| \geq 1$ for $|x|=1$. By (5.3), this polynomial dominates $x^{k-1}+1$ on the unit circle, with equality only at the roots of $\left(x^{k}+1\right)(x-1)$. Thus, again by Rouché's theorem applied to the numerator of the right member of $(5.2), P_{k}(x)$ is a Pisot polynomial multiplied possibly by a factor of $\left(x^{k}+1\right)(x-1)$. This factor is easily seen to be $x-1$ if $k$ is even and $x^{2}-1$ if $k$ is odd.

(2) As in the proof of Proposition 5.1, it is clear that $20^{k}: 01^{k-1} 01^{k-2} 0110^{k-2} 1$ is the expansion of 1 for some $\beta>1$. A little calculation shows that the characteristic polynomial $R_{k}(x)$ for this expansion is given by

$(x-1) R_{k}(x)=x^{4 k+1}(x-1)(x-2)-x^{3 k}+x^{2 k}(x-1)+2 x^{k}(x-1)+x^{k-1}-x+1$.

It now suffices to verify that $P_{k}(x) Q_{k}(x)=(x-1) R_{k}(x)$, an elementary exercise (which is made even easier by Maple).

(3) Clearly, $Q_{k}(x)=x^{2 k+2}-x^{2 k+1}+x^{k+1}-x+1$ is reciprocal, as is $(x+1) Q_{k}(x)$. To show that it is noncyclotomic for sufficiently large $k$, we compute its Mahler measure

$$
M\left(Q_{k}\right)=\prod_{\gamma} \max (|\gamma|, 1)=\exp \left(\int_{0}^{1} \log \left|Q_{k}(e(t))\right| d t\right),
$$

where $\gamma$ runs over the zeros of $Q_{k}$, and where $e(t)=\exp (2 \pi i t)$. So $M\left(Q_{k}\right)=1$ if and only if $Q_{k}$ is cyclotomic. By a result of [9], generalized in [20], if $F(x, y)$ is a Laurent polynomial in two variables, then

$$
\lim _{k \rightarrow \infty} M\left(F\left(x, x^{k}\right)\right)=M(F(x, y))
$$

where $M(F(x, y))$ is the geometric mean over the 2-torus of $|F(x, y)|$, i.e.,

$$
\log M(F(x, y))=\int_{0}^{1} \int_{0}^{1} \log |F(e(s), e(t))| d s d t .
$$

Thus,

$$
\lim _{k \rightarrow \infty} M\left(Q_{k}\right)=M\left(y^{2}-y^{2} x^{-1}+y-x+1\right) .
$$

By the change of variables $-x / y \rightarrow x$, we see that

$$
M\left(y^{2}-x^{-1} y^{2}+y-x+1\right)=M(x+y+1+1 / y+1 / x)=1.28573 \ldots,
$$

one of the limit points of Mahler measures mentioned in [9]. Since $\lim M\left(Q_{k}\right)>1$, $Q_{k}$ can be cyclotomic only for finitely many $k$. (Computation suggests that $Q_{k}$ is cyclotomic only for $k=1,2$ and 3. An estimate similar to that given in [9, Appendix 2] should suffice to establish this, since it is easy to verify by computation that it is true for $k \leq 100$.) 
Sketch of Proofs of Propositions 5.3-5.5. We will not present the full proofs of these results here. In broad outline, the proofs follow those of Propositions 5.1 and 5.2. One determines the beta-expansion by inspection and then proves that it is correct using Parry's criterion and elementary polynomial algebra (assisted in all cases by Maple). The restrictions $k \geq 4$ and $k \geq 3$ in Propositions 5.3 and 5.4 arise at this point in the proof.

The main difficulty is the proof that $P_{k}(x)$ has exactly one root outside the unit circle. Our method of proof is suggested by similar considerations in [6].

For Proposition 5.3, we begin with the observation that if $P_{k}^{*}(x)=x^{5 k+2} P_{k}(1 / x)$ is the reciprocal of $P_{k}$, then $P_{k}+P_{k}^{*}=-x^{5 k+1}-x$, whose roots are 0 and the $5 k$ th roots of -1 . Let $x_{j}(t), 1 \leq j \leq 5 k+2$, be the roots of $f(x, t):=P_{k}(x)+t P_{k}(x)=0$, where, say $x_{1}(1), \ldots, x_{5 k}(1)$ are the roots of $x^{5 k}+1, x_{5 k+1}(1)=0$ and $x_{5 k+2}=\infty$. By the symmetry of $f(x, t)$ under $x \rightarrow 1 / x$, no $x_{j}(t)$ lies on the unit circle for $0 \leq t<1$ unless it is a common root of $P_{k}$ and $P_{k}^{*}$ (these roots account for the factor $\left.T_{k}(x)\right)$. This symmetry also shows that the curves $x_{j}(t)$ are orthogonal to the unit circle at $t=1$ (cf. $[6, \S 3])$.

The roots of $P_{k}(x)=0$ are $x_{j}(0), j=1, \ldots, 5 k+2$. Clearly, $\left|x_{5 k+1}(0)\right|<1$ and $x_{5 k+2}(0)>1$, so to show that $P_{k}(x) / T_{k}(x)$ is a Pisot polynomial, it suffices to show that the tangent to the curve $x_{j}(t)$ at $t=1$ points out of the unit circle, i.e., that $\frac{d x_{j}}{d t}(1) / x_{j}(1)>0$ for $j=1, \ldots, 5 k$. This reduces to showing that $\omega^{-1} P_{k}(\omega)>0$ for each root $\omega$ of $x^{5 k}+1$ that is not a root of $T_{k}(x)$. This follows by simple estimates, once one distinguishes the five possible values of $\omega^{k}$.

Similar proofs apply to the $P_{k}(x)$ of Propositions 5.4 and 5.5. For example, for Proposition 5.4, $P_{k}(x)-P_{k}^{*}(x)=-x\left(x^{8 k}-1\right) /\left(x^{2 k}+1\right)$, which has roots on the unit circle at some of the $8 k$ th roots of 1 .

Remark 5.4. In addition to the families described in Propositions 5.3 - 5.5, we have found a number of other examples of the same type with $\beta_{k}<2$ for which the quantities $m$ and $p$ depend linearly on $k$ and for which $Q_{k}$ is nonreciprocal. An apparently similar example with a very different behavior is the following:

$$
\begin{aligned}
P_{k}(x)=x^{6 k+1}( & x-2)+x^{5 k+1}(x-1)+x^{4 k}(x-1) \\
& -x^{3 k}\left(x^{2}-2 x+1\right)-x^{2 k+1}(x-1)-x^{k}(x-1)-(x-1) .
\end{aligned}
$$

The values of $k, m, p$ and the type of $Q_{k}$ for $1 \leq k \leq 20$ are given in Table 2 . Note that we did not compute the polynomials $Q_{k}$ for $18 \leq k \leq 20$ but verified that they were noncyclotomic by computing a single root outside the unit circle directly from the beta-expansions. Although the period lengths for $k=5,6,7,8,9$ and 14 are given by $p=6 k$, the periods (i.e. the words $c_{m+1} \ldots c_{m+p}$ ) themselves do not follow a common pattern. We can prove that $P_{k}$ defines a Pisot number for each $k \geq 1$ by the method of Propositions 5.3-5.5. However, we do not know how to prove that the unusual behavior of the beta-expansions indicated in Table 2 for $k \leq 20$ will persist for larger $k$.

Remark 5.5. In all of these examples $\beta_{k}$ satisfies a polynomial of the form $f\left(x, x^{k}\right)$, where $f(x, y)$ is a polynomial in two variables. The proofs that $f\left(x, x^{k}\right)$ has exactly one root outside the unit circle seem somewhat ad hoc. The general question to which one is led is to find the exact conditions on a polynomial $f(x, y) \in \mathbb{C}[x, y]$ 
TABLE 2 (see Remark 5.4)

\begin{tabular}{cccc}
$k$ & $m$ & $p$ & type \\
\hline 1 & 2 & 6 & $\mathrm{C}$ \\
2 & 7 & 45 & $\mathrm{C}$ \\
3 & 10 & 48 & $\mathrm{C}$ \\
4 & 55 & 49 & $\mathrm{NR}$ \\
5 & 237 & 30 & $\mathrm{NR}$ \\
6 & 395 & 36 & $\mathrm{NR}$ \\
7 & 861 & 42 & $\mathrm{NR}$ \\
8 & 2117 & 48 & $\mathrm{NR}$ \\
9 & 856 & 54 & $\mathrm{NR}$ \\
10 & 2314 & 566 & $\mathrm{NR}$ \\
11 & 2190 & 113 & $\mathrm{NR}$ \\
12 & 1530 & 578 & $\mathrm{NR}$ \\
13 & 1979 & 625 & $\mathrm{NR}$ \\
14 & 4086 & 84 & $\mathrm{NR}$ \\
15 & 3080 & 796 & $\mathrm{NR}$ \\
16 & 2755 & 930 & $\mathrm{NR}$ \\
17 & 2633 & 3428 & $\mathrm{NR}$ \\
18 & 2427 & 55648 & not C \\
19 & 3030 & 219156 & not C \\
20 & 5229 & 337160 & not C
\end{tabular}

under which $f\left(x, x^{k}\right)$ has a single root outside the unit circle for all sufficiently large $k$. We hope to return to a discussion of this interesting question in a future paper.

\section{REFERENCES}

1. M. Amara, Ensembles fermés de nombres algébriques, Ann. Sci. École Norm. Sup. (3) 83 (1966), 215-270. MR 38:5741

2. D. Berend and C. Frougny, Computability by finite automata and Pisot bases, Math. Systems Theory 27 (1994), 275-282. MR 95a:11109

3. M.J. Bertin, A. Decomps-Guilloux, M. Grandet-Hugot, M. Pathiaux-Delefosse, and J.P. Schreiber, Pisot and Salem numbers, Birkhäuser Verlag, Basel, 1992. MR 93k:11095

4. A. Bertrand, Développements en base de Pisot et répartition modulo 1, C.R. Acad. Sci. Paris Sér. I Math. 285 (1977), 419-421. MR 56:5449

5. A. Bertrand-Mathis, Développement en base $\theta$, répartition modulo un de la suite $\left(x \theta^{n}\right)_{n \geq 0}$, langages, codes et $\theta$-shift,, Bull. Soc. Math. France 114 (1986), 271-323. MR 88e:11067

6. D.W. Boyd, Small Salem numbers, Duke Math. J. 44 (1977), 315-328. MR 56:11952

7. _ Pisot and Salem numbers in intervals of the real line, Math. Comp. 32 (1978), 1244-1260. MR 58:10812

8. _ Reciprocal polynomials having small measure, Math. Comp. 35 (1980), 1361-1377. MR 82a:30005

9. _ Speculations concerning the range of Mahler's measure, Canad. Math. Bull. 24 (1981), 453-469. MR 83h:12002

10. _ Pisot numbers in the neighbourhood of a limit point. I, J. Number Theory 21 (1985), 17-43. MR 87c:11096a

11. _ Pisot numbers in the neighborhood of a limit point. II, Math. Comp. 43 (1984), 593-602. MR 87c:11096b 
12. Salem numbers of degree four have periodic expansions, Théorie des Nombres Number Theory (J.M. de Koninck and C. Levesque,, eds.), de Gruyter, Berlin and New York, 1989, pp. 57-64. MR 90j:11071

13. R.J. Bradford and J.H. Davenport, Effective tests for cyclotomic polynomials, Lecture Notes in Comput. Sci., vol. 358, Springer-Verlag, Berlin and New York, 1989, pp. 244-251. MR 90m:11201

14. B.W. Char, K.O. Geddes, G.H. Gonnet, B.L. Leong, M.B. Monagan, and S.M. Watt, Maple V Language Reference Manual, Springer-Verlag, Berlin and New York, 1991.

15. J. Dufresnoy and Ch. Pisot, Étude de certaines fonctions méromorphes bornées sur le cercle unité, application à un ensemble fermé d'entiers algébriques, Ann. Sci. École Norm. Sup. (3) $\mathbf{7 2}$ (1955), 69-92. MR 17:349d

16. Sur les éléments d'accumulation d'un ensemble fermé d'entiers algébriques, Bull. Sci. Math. (2) 79 (1955), 54-64. MR 17:463a

17. L. Flatto, J.C. Lagarias, and B. Poonen, The zeta function of the beta transformation, Ergodic Theory Dynamical Systems 14 (1994), 237-266. MR 95c:58141

18. C. Frougny and B. Solomyak, Finite $\beta$-expansions, Ergodic Theory Dynamical Systems 12 (1992), 713-723. MR 94a:11123

19. A.O. Gelfond, On a general property of number systems, Izv. Akad. Nauk SSSR Ser. Mat. 23 (1959), 809-814. (Russian) MR 22:702

20. W. Lawton, A problem of Boyd concerning geometric means of polynomials, J. Number Theory 16 (1983), 356-362. MR 84i:10056

21. W. Parry, On the $\beta$-expansions of real numbers, Acta Math. Hungar. 11 (1960), 401-416. MR 26:288

22. R. Salem, A remarkable class of algebraic integers. Proof of a conjecture of Vijayaraghavan, Duke Math. J. 11 (1944), 103-108. MR 5:254a

23. K. Schmidt, On periodic expansions of Pisot numbers and Salem numbers, Bull. London Math. Soc. 12 (1980), 269-278. MR 82c:12003

24. B. Solomyak, Conjugates of beta-numbers and the zero-free domain for a class of analytic functions, Proc. London Math. Soc. (3) 68 (1994), 477-498. MR 95c:30010

25. F. Talmoudi (= F. Lazami Talmoudi), Sur les nombres de $S \cap[1,2]$, C.R. Acad. Sci. Paris Sér. Math. 285 (1977), 969-971. MR 80c:12003

26. _ Sur les éléments de $S \cap[1,2[$, C. R. Acad. Sci. Paris Sér. Math. 287 (1978), 739-741. MR 82a: 12001

Department of Mathematics, University of British Columbia, Vancouver, B.C., CANADA V6T $1 \mathrm{Z2}$

E-mail address: boyd@math.ubc.ca 